\title{
Effects of High-Intensity Training on Anaerobic and Aerobic Contributions to Total Energy Release During Repeated Supramaximal Exercise in Obese Adults
}

\author{
Georges Jabbour ${ }^{*}$, Horia-Daniel lancu and Anne Paulin
}

\begin{abstract}
Background: Studying relative anaerobic and aerobic metabolism contributions to total energy release during exercise may be valuable in understanding exercise energetic demands and the energetic adaptations that occur in response to acute or chronic exercise in obese adults. The aim of the present study is to evaluate the effects of 6 weeks of high-intensity training (HIT) on relative anaerobic and aerobic contributions to total energy release and on peak power output during repeated supramaximal cycling exercises (SCE) in obese adults.

Methods: Twenty-four obese adults (body mass index $= \pm 33 \mathrm{~kg} \cdot \mathrm{m}^{-2}$ ) were randomized into a control group $(n=12)$ and an HIT group $(n=12)$. Accumulated oxygen deficits $\left(\mathrm{ml}^{\mathrm{min}}{ }^{-1}\right)$ and anaerobic and aerobic contributions (\%) were measured in all groups before and after training via repeated SCE. In addition, the peak power output performed during SCE was determined using the force-velocity test.

Results: Before HIT, anaerobic contributions to repeated SCE did not differ between the groups and decreased significantly during the third and fourth repetitions. After HIT, anaerobic contributions increased significantly in the HIT group ( $+11 \%, p<0.01)$ and were significantly higher than those of the control group $(p<0.01)$. Moreover, the peak

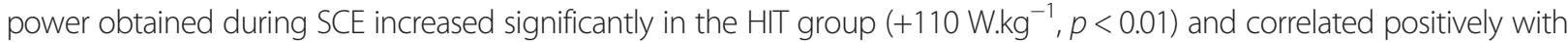
increases in anaerobic contributions $(r=0.9, p<0.01)$.

Conclusions: In obese adults, HIT increased anaerobic contributions to energy release which were associated with peak power enhancement in response to repeated SCE. Consequently, HIT may be an appropriate approach for improving energy contributions and muscle power among obese adults.
\end{abstract}

Keywords: High-intensity training; Aerobic; Anaerobic; Energy contribution

\section{Key Points}

- Anaerobic contributions to energy release increased significantly in the trained group following HIT. These higher anaerobic contributions were accompanied by both greater AOD deficits and larger decreases in oxygen uptake during SCE repetitions.

- HIT may be an appropriate approach for enhancing the level of substrate phosphorylation from $\mathrm{PCr}$ hydrolysis and glycolysis during repeated supramaximal exercise bouts, which may be partially related to enhancements in the muscle efficacy of obese adults.

- In obese adults, studying relative anaerobic and aerobic metabolism contributions to total energy release during exercise may be valuable in understanding exercise energetic demands and the energetic adaptations that occur in response to acute or chronic exercise.

* Correspondence: georgesjabbour1980@hotmail.com

School of Kinesiology and Leisure, Faculty of Health Sciences and

Community Services, Université de Moncton, Moncton, NB E1A 3E9, Canada

\section{实 Springer}

(C) 2015 Jabbour et al. Open Access This article is distributed under the terms of the Creative Commons Attribution 4.0 International License (http://creativecommons.org/licenses/by/4.0/, which permits unrestricted use, distribution, and reproduction in any medium, provided you give appropriate credit to the original author(s) and the source, provide a link to the Creative Commons license, and indicate if changes were made. 


\section{Background}

Several studies have recently focused on determining energetic system contributions in several sports practices, with the objective of understanding the energetic demand related to these events in order to develop improved training recommendations $[1,2]$. In the context of obesity, exercise energy metabolism is impaired [3, 4]. Aerobic energy release during peak exercise is reduced by excessive fat mass, which imposes an unfavorable burden on cardiac function and on oxygen uptake by working muscles [5]. Evaluation of aerobic energy release has become part of routine clinical assessments of this population, as the degree of its reduction is a strong predictor of an individual's health [6]. By contrast, anaerobic metabolic pathways have received less attention. Studies involving obese individuals are more focused on evaluating anaerobic aptitude (e.g., maximal power output). These studies describe lower values of peak power output in obese subjects compared with normal-weight subjects $[7,8]$. This reduced parameter has been linked to excessive fat mass, which may reduce motor-unit activation and the ratio of muscle strength to units of muscle mass [8]. However, no data are available regarding anaerobic energetic yield during exercise. Therefore, studying energetic muscle interactions for a given exercise may be the best approach to describing the true metabolic energy of the muscles of obese individuals and understanding the metabolic adaptations that occur in response to an exercise intervention in obese individuals.

Regarding intervention strategies and their impact on metabolic profiles, high-intensity training, such as supramaximal exercise, is gaining popularity in the context of obesity management. Based on data provided by postintervention metabolic measures, this model of exercise appears to be a valuable approach for managing obesity [9]. Indeed, Whyte et al. [10] demonstrated that very high-intensity sprint interval training [2 weeks, comprising 6 sessions of 4 to 6 repeats of 30-s Wingate anaerobic sprints on an electromagnetically braked cycle ergometer, with a 4.5-min recovery period between each repetition] improved several metabolic risk factors: 1) an increase in resting fat oxidation rate in the fasted state and 2) a decrease in resting carbohydrate oxidation in the fasted state compared with baseline. This highintensity training also decreased waist and hip circumferences in overweight and obese sedentary men and illustrated the potential of this alternative exercise model to improve lipid utilization in individuals with excess body weight. However, no information describing true metabolic adaptations during exercise is available. More importantly, a simultaneous evaluation of the maximal activation of both energetic systems (e.g., anaerobic vs. aerobic) may allow us to better understand anaerobic/aerobic interactions during exercise and provide information regarding the factor(s) that limit performance in obese individuals, as well as for other populations (normal-weight individuals, athletes).

Accumulated oxygen deficit (AOD) is a descriptor of the maximal amount of energy obtained from anaerobic metabolism [11] and makes it possible to directly measure maximal anaerobic energy release during supramaximal effort. This concept makes it possible to simultaneously evaluate the maximal activation of both anaerobic and aerobic metabolic systems and obtain a complete and coherent description of exercise energy demands in obese individuals. Based on the Oxygen consumption $\left(\mathrm{VO}_{2}\right)$ predicted from the individual $\mathrm{VO}_{2}-$ intensity relationship determined from the graded exercise test, the AOD can be determined during supramaximal exercise protocol [12]. It has been admitted that AOD measures are generally accepted as the criterion measure of anaerobic energy expenditure as a result of flaws in the use of blood lactate concentration as a quantitative tool for the measurement of anaerobic energy supply [13].

The aim of the present study is to evaluate the relative anaerobic and aerobic contributions to total energy release during repeated bouts of supramaximal cycling exercise in obese adults undergoing 6 weeks of supramaximal exercise training. Therefore, the examination of muscle metabolism and power output profiles in response to repeated bouts of cycling sprinting with a short recovery interval may enable us to better understand the regulation of these metabolic pathways.

\section{Methods}

\section{Experimental Subjects}

Twenty-four young adults (13 women and 11 men) were recruited from the Moncton campus of the University of Moncton. To do so, we invited by posting announcement to voluntarily participate students of the University of Moncton who applied the inclusion criteria. The study protocol was approved by the University's Human Research Ethics Committee (UHRC), and all procedures followed were in accordance with the Helsinki Declaration of 1975, as revised in 2008. Informed consent was obtained from all patients for being included in the study. In addition to being obese, the inclusion criteria for participation were as follows: participants had to be physically active fewer than 60 min.week $^{-1}$ as assessed by the International Physical Activity Questionnaire [14] and have no history of metabolic, cardiovascular, or chronic health problems, no history of drug consumption before the study, and no history of smoking. Before entering our protocol, each of the participants was thoroughly familiarized with all testing equipment and procedures. Indeed, each subject cycled for an extended period of time on the same cycle ergometer used throughout the study. Additionally, each participant 
was asked to determine the height of seat at which they are able to pedal comfortably. Unfortunately, we do not have any objective positioning (knee angle, hip angle, etc.) and this may be considered as limitation of the study. But, it is important to note that the position of each participant, e.g., the seat height, was the same throughout the study.

The protocol then began with three sessions of preliminary testing in order to determine certain key variables. The testing was conducted on three different days (D1, D2, and D3) after an overnight fast. Each day was separated by a minimum of $48 \mathrm{~h}$, and all subjects were asked to avoid physical activity for $48 \mathrm{~h}$ prior to each session. In addition, subjects were asked to be well hydrated before each test. The same protocol that has been previously developed by our laboratory [15] seems suitable to delay any early fatigue or discomfort among participants which were all sedentary.

Following the determination of body composition, obese participants (BMI $>30 \mathrm{~kg} / \mathrm{m}^{2}$ ) were selected based on the Canadian guidelines for body weight classification in adults [16] and were separated in the following two groups: a control group (without any intervention; $n=12$ ) and a training group $(n=12)$. Fat-free mass was calculated by subtracting fat mass from body mass.

\section{Anthropometric Measurements}

Body mass was measured to the nearest $0.1 \mathrm{~kg}$ with the subject in light clothing, without shoes, using an electronic scale (Kern, MFB 150 K100). Height was determined to the nearest $0.5 \mathrm{~cm}$ with a measuring tape fixed to the wall. Body mass index (BMI) was calculated as the ratio of mass $(\mathrm{kg})$ to height ${ }^{2}\left(\mathrm{~m}^{2}\right)$. Body fat percentage was estimated using a bioimpedance machine (Vacumed, Bodystat1500).

\section{Physiology Assessment}

On day 1 (D1), subjects performed a maximal test on an upright cycle ergometer (Monark ergomedic 839E electronic test cycle, USA) to determine their peak oxygen consumption $\left(\mathrm{VO}_{2 \text { peak }}\right)$. Before beginning the test, adults remained seated for $5 \mathrm{~min}$ on the bicycle ergometer in the same position used in subsequent exercise. Resting oxygen consumption was measured based on the mean oxygen consumption of the last $30 \mathrm{~s}$ of minutes 3,4 , and 5 . No proper warm-up was performed. The test started at an initial power of $25 \mathrm{~W}$ and was progressively increased by $25 \mathrm{~W}$ every $2 \mathrm{~min}$ until exhaustion. In the present study, all participants reach exhaustion at $125 \mathrm{~W}$. In fact, those who could not achieve a power output greater than or equal to $125 \mathrm{~W}$ were excluded.

A breath-by-breath automated metabolic system (CPX, Medical Graphics, St. Paul, Minnesota, USA) was used to determine the $\mathrm{VO}_{2 \text { peak }}$ of each participant. Calibration prior to each test was performed with standard gases of known oxygen and carbon dioxide concentration for gas composition and a calibration syringe for volume. The data were averaged on a 30-s interval, and oxygen uptake and respiratory exchange ratio, which is the ratio of carbon dioxide produced to oxygen consumed, were obtained.

Peak oxygen consumption was achieved when a subject fulfilled at least three of the following criteria: a plateau in $\mathrm{VO}_{2}$ in spite of an increase in exercise intensity, a respiratory exchange ratio greater than 1.1, a maximal HR above $90 \%$ of the predicted maximal theoretical HR (220-age in years) or apparent exhaustion [17].

On day 2 (D2), we measured steady-state $\mathrm{VO}_{2}$ uptake at a constant submaximal power (below the $\mathrm{VO}_{2 \text { peak }}$ ). After a 3-min resting baseline period, subjects started pedaling at the established power, which was maintained for $10 \mathrm{~min}$ at a pedaling rate of 60 revolutions $/ \mathrm{min}(\mathrm{rpm})$. The 10-min exercise bouts were completed 6 times for each subject at powers of $30,40,50,60,70$, and $80 \%$ of their individual $\mathrm{VO}_{2 \text { peak }}$ power and were separated by a 5 -min resting period, and the measurements for each subject were plotted separately and visually checked for linearity as described elsewhere [18]. This demonstrates that our measured $\mathrm{VO}_{2}$ uptake for a 10 -min exercise was a steady-state value equaling $\mathrm{VO}_{2}$ demand (total rate of energy release). We therefore conclude that $\mathrm{VO}_{2}$ demand increased linearly with power for all subjects in the examined range. This analysis allowed for the calculation of the accumulated oxygen deficit (AOD) (measured in $\mathrm{ml} \mathrm{O}_{2}$ equivalents per $\mathrm{kg}$ ) for each cycling interval by calculating the difference between $\mathrm{VO}_{2}$ demand for the respective power (from extrapolation of the calculated relationship) and $\mathrm{VO}_{2}$ uptake. The linear relationship between steady-state $\mathrm{VO}_{2}$ values and cycling power was extrapolated and used to estimate energy demand during supramaximal cycling exercise (SCE) [19].

On day 3 (D3), following $10 \mathrm{~min}$ of warm-up, subjects performed a force-velocity test on a cycle ergometer using a technique adapted from the study performed by Vandewalle et al. [20]. This test consists of a succession of supramaximal bouts of approximately $6 \mathrm{~s}$, with exercise loads increasing by $1 \mathrm{~kg}$ after each bout until the subject is unable to perform the test. A period of passive recovery $(5 \mathrm{~min})$ was allowed between successive bouts. The peak velocity for each bout was recorded, and the power output was calculated by multiplying the load and speed. The optimal load corresponded to the load at which maximal power $\left(\mathrm{PO}_{\max }\right)$ was achieved. This load was then used for the training protocol that followed. The force-velocity test was also performed every 2 weeks to adjust the individual power level of $\mathrm{SCE}$. These 3 days of testing were completed before high-intensity training (HIT) (visit 1), and also at the end of training (visit 2). 


\section{Training Session}

Once participants completed preliminary testing, they were instructed to complete a total of 18 training sessions (three sessions per week for 6 weeks). Each of the prescribed sessions began with a 5-min warm-up of continuous cycling at moderate intensity ( $40 \%$ of their individual $\mathrm{VO}_{2 \text { peak }}$ power), followed by 6 repetitions of SCE intervals with 2 min of passive recovery between each repetition. Each SCE repetition lasted $6 \mathrm{~s}$, and participants were asked to pedal at maximal velocity against the resistance determined during D3. The repeat sprint cycling test was conducted under the supervision of a member of the research team, and velocities (in RPM) were recorded for each second of the bout in order to ensure that said velocities were constant. Based on the linear regression and the individual $\mathrm{VO}_{2 \mathrm{max}}$, the workload approximately corresponded to $\sim 350 \%$ of $\mathrm{VO}_{2 \max }$ [12].

In fact, we have chosen in the present study the HIT for many reasons. Firstly, very brief high-intensity repeated exercise comprised of 6- to 10-s sprints induces substantial improvements in both performance and health-related outcomes [21]. For example, 2 to 15 weeks of this type of training results in significant increases in anaerobic capacity, ranging between 5 and $28 \%$ in untrained males [21]. Moreover, it represents a timeefficient approach to obtain health benefits from exercise, as sessions involve a total of only 2-3 min of high-intensity exercise and typically last for less than $15 \min [22,23]$.

Training sessions were conducted under the supervision of a member of the research team, and velocities (in RPM) were recorded for each second of the bout in order to ensure that said velocities were constant. Additional testing was also completed during the first prescribed training session, as well as during the training session at the end of the sixth week. Indeed, during the first and the final sessions, participants were asked to perform one of their regular training sessions while being analyzed by our breath-by-breath automated metabolic system during only four repetitions. This was done in order to determine the contribution of each energy system. In this study, results are given only from four supramaximal cycling exercise bout given that some of participants felt discomfort by wearing mask destined for gas analysis. Then, the mask was removed to allow participants to complete the entire session bouts. Lactate concentrations were obtained at rest for all experimental subjects, and immediately following the fourth repetition, via capillary blood samples using the LactatePro analyzer. This technique was previously utilized in our laboratory to monitor athletes. In the present study, lactate concentrations served only to complete the profiles of the control and training groups, before and after HIT.

After completing 6 weeks of training, participants were asked to return for a final day of testing (visit 2), during which the procedures of D1, D2, and D3 were repeated, and post-training data was collected.

\section{Calculation of Relative Energy Expenditure: Supramaximal Cycling Exercise Bout}

The $\mathrm{VO}_{2}$ demand values of SCE were estimated individually by extrapolating the linear relationship between the power and the $\mathrm{VO}_{2}$ demand values established during the constant submaximal power exercises. The accumulated $\mathrm{VO}_{2}$ uptake and the accumulated $\mathrm{VO}_{2}$ demand were taken as the $\mathrm{VO}_{2}$ uptake and the $\mathrm{VO}_{2}$ demand integrated over the entire supramaximal cycling exercise bout [18]. The $\mathrm{AOD}$ was equal to the accumulated $\mathrm{VO}_{2}$ demand minus the accumulated $\mathrm{VO}_{2}$ uptake. This allowed for a measurement of anaerobic (AOD) and aerobic $\left(\mathrm{VO}_{2}\right)$ energy contributions throughout the four SCE repetitions [24].

\section{Statistical Analyses}

After testing for normality (Kolmogorov-Smirnov test), statistical comparisons were made between the control group and the training group on two separate occasions (before and after training). Two-way repeated measures ANOVA was used to determine whether significant changes in energy system contributions emerged between the two groups, and if energy system contributions differed between the two groups. Furthermore, Bonferroni's post hoc test was performed. Pearson correlations were used to assess the relationship among changes in muscle power, aerobic capacity, and energy contribution modification. A value of $p<0.05$ was statistically significant. Analyses were performed using IBM SPSS Statistics 19 software.

\section{Results}

Height, body mass, body mass index, fat mass, and fatfree mass were similar across the two groups during visit 1 and visit 2 (Table 1 ). Resting heart rates were lower for the trained group during visit 2 compared with visit $1(p<0.01)$ and were also lower than those of the control group during visit $2(p<0.01) . \mathrm{VO}_{2 \max }$ values assessed after 6 weeks of HIT training did not differ from those of visit 1 and visit 2 . The maximal power output obtained during the force-velocity test increased significantly in the training group compared with visit 1 and was significantly higher than the corresponding values of the control group for visit 1 and visit 2 (Table 1 ).

During all SCE bouts, heart rate values did not differ among the groups (Table 2). For the trained group, the power output exhibited during SCE was significantly higher compared with that observed during visit $1(p<.0 .1)$ and was significantly different compared with that of the control group during visit $1(p<0.01)$ and visit $2(p<0.01)$, and compared with the fourth repetitions. $\mathrm{VO}_{2}$ uptake did not 
Table 1 Age, anthropometric, aerobic and anaerobic fitness parameters of young obese adults at visit 1 and visit 2

\begin{tabular}{|c|c|c|c|c|c|c|}
\hline & \multicolumn{2}{|l|}{ Visit 1} & \multicolumn{2}{|l|}{ Visit 2} & \multirow{2}{*}{$\begin{array}{l}\Delta \text { Visit } 2 \text { vs. visit } 1 \\
\text { for control group }\end{array}$} & \multirow{2}{*}{$\begin{array}{l}\Delta \text { Visit } 2 \text { vs. visit } \\
\text { for trained group }\end{array}$} \\
\hline & $\begin{array}{l}\text { Control } \\
(n=12) \\
(w=7, m=5)\end{array}$ & $\begin{array}{l}\text { Trained } \\
(n=12) \\
(w=6, m=6)\end{array}$ & $\begin{array}{l}\text { Control } \\
(n=12) \\
(w=7, m=5)\end{array}$ & $\begin{array}{l}\text { Trained } \\
(n=12) \\
(w=6, m=6)\end{array}$ & & \\
\hline \multicolumn{7}{|l|}{ Age and anthropometrics } \\
\hline Age (year) & $23.1(3.3)$ & $22.5(2.3)$ & $23.3(2.3)$ & $22.7(2.2)$ & 0.2 & 0.2 \\
\hline Height (cm) & $1.71(0.11)$ & $1.74(0.09)$ & $1.71(0.11)$ & $1.74(0.09)$ & - & - \\
\hline Body mass (kg) & $99.5(24.1)$ & $101.1(21.1)$ & $100.5(21.1)$ & $99.9(9.1)$ & 1 & -1.1 \\
\hline BMI $\left(\mathrm{kg} \cdot \mathrm{m}^{-2}\right)$ & $33.3(4.8)$ & $33.2(2.8)$ & $33.7(3.8)$ & $33.1(3.7)$ & 0.4 & -0.1 \\
\hline FM (\%) & $42.3(9.4)$ & $42.8(7.4)$ & $44.3(9.4)$ & $42.1(7.1)$ & 2 & -0.6 \\
\hline FFM (kg) & $51.2(10.1)$ & $50.2(9.1)$ & $50.2(9.1)$ & $50.8(5.7)$ & -1 & 0.5 \\
\hline \multicolumn{7}{|c|}{ Aerobic and anaerobic fitness indicators } \\
\hline HR peak (beats.min ${ }^{-1}$ ) & $196(12)$ & $196(09)$ & $197(16)$ & $198(12)$ & 1 & -2 \\
\hline RERpeak & $1.1(0.1)$ & $1.1(0.3)$ & $1.1(0.3)$ & $1.1(0.2)$ & - & - \\
\hline$P_{\text {VO2max }}(W)$ & $125(0.1)$ & $125(0.1)$ & $125(0.1)$ & $125(0.1)$ & - & - \\
\hline $\mathrm{VO}_{2 \max }\left(\mathrm{ml} \cdot \mathrm{min}^{-1} \cdot \mathrm{kg}^{-1}\right)$ & $23.4(8.4)$ & $22.6(6.4)$ & $22.2(7.4)$ & $23.1(6.1)$ & -1.2 & 0.5 \\
\hline $\mathrm{PO}_{\max }(\mathrm{W})$ & $470(30)$ & $465(35)$ & $465(25)$ & $570(40)^{*, * *}$ & -5 & 105 \\
\hline
\end{tabular}

Values are expressed as the mean (standard deviation)

$w$ woman, $m$ men, $B M I$ body mass index, FM fat mass, FFM fat-free mass, $H R$ heart rate, $R E R$ respiratory exchange ratio, $P_{V O 2 m a x}$ maximal power output obtained during the maximal test, $V \mathrm{O}_{2 \max }$ maximal oxygen consumption, $P \mathrm{O}_{\max }$ maximal power output developed during the force-velocity test, $\Delta$ variation between parameters assessed at visit 1 and visit 2

*Significant difference between groups $(p<0.01) ;{ }^{* *}$ significant difference from visit $1(p<0.01)$

differ statistically across visits for the control group. However, following HIT, the $\mathrm{VO}_{2}$ uptake values determined during the first, second, third, and fourth repetitions decreased significantly in the trained group $(p<0.01)$ and were significantly lower than the values of the control group obtained during visit $1(p<0.01)$ and visit $2(p<0.01)$ (Table 2). For both the control group and the trained group, $\mathrm{VO}_{2}$ uptake increased significantly during the third and fourth repetitions compared with values obtained during the first repetition, before HIT $(p<0.01)$ (Table 2). By contrast, the AOD decreased for the trained group before HIT, and for the control group during the third and foruth repetitions $(p<0.01)$. Following HIT, the trained group demonstrated significantly higher AOD values compared with those obtained before HIT $(p<0.01)$, and compared with the control group $(p<0.01)$. Additionally, the $\mathrm{VO}_{2}$ uptake, the AOD, the aerobic, and the aerobic contributions determined following HIT remained constant during all repetition bouts (Table 2).

Anaerobic energy contributions (calculated using the AOD method) increased significantly for the training group following HIT $(p<0.01)$ and were also significantly higher during SCE during the first, second, third, and fourth repetitions compared with values obtained for the control group during visit $1(p<0.01)$ and visit 2 $(p<0.01)$. During the third and the fourth repetitions, the control group and the trained group demonstrated significant decreases in anaerobic contributions compared with values obtained during the first repetition, decreases that were accompanied by increases in the values of the aerobic contributions, before HIT (Table 2).

Lactate concentrations measured at rest did not differ between the groups during visit 1 and visit 2 . Following HIT, lactate concentrations increased significantly in the trained group compared with visit $1\left(\sim 6\right.$ mmol.kg $\left.{ }^{-1}, p<0.01\right)$ and were significantly higher than those observed for in the control group during visit 1 and visit $2(p<0.01)$.

In this study, the increased power output levels observed for the trained group following HIT and during the SCE bouts correlated positively with anaerobic contribution increases $(r=0.9, p<0.01$, respectively). However, no relationships were observed between anaerobic contribution increases and $\mathrm{VO}_{2 \max }(r=0.08, p=0.7)$.

\section{Discussion}

To the best of our knowledge, this study is the first to examine the effects of HIT on relative anaerobic and aerobic contributions to total energy release in obese adults during repeated bouts of supramaximal cycling exercise. The primary finding of this study was that relative anaerobic and aerobic contributions to total energy release during repeated bouts of SCE differed in untrained obese adults compared with the obese group following HIT. Indeed, anaerobic contributions to energy release increased significantly in the trained group following HIT. These higher anaerobic contributions 
Table 2 Calculated and measured variables during the supramaximal cycling exercise (SCE)

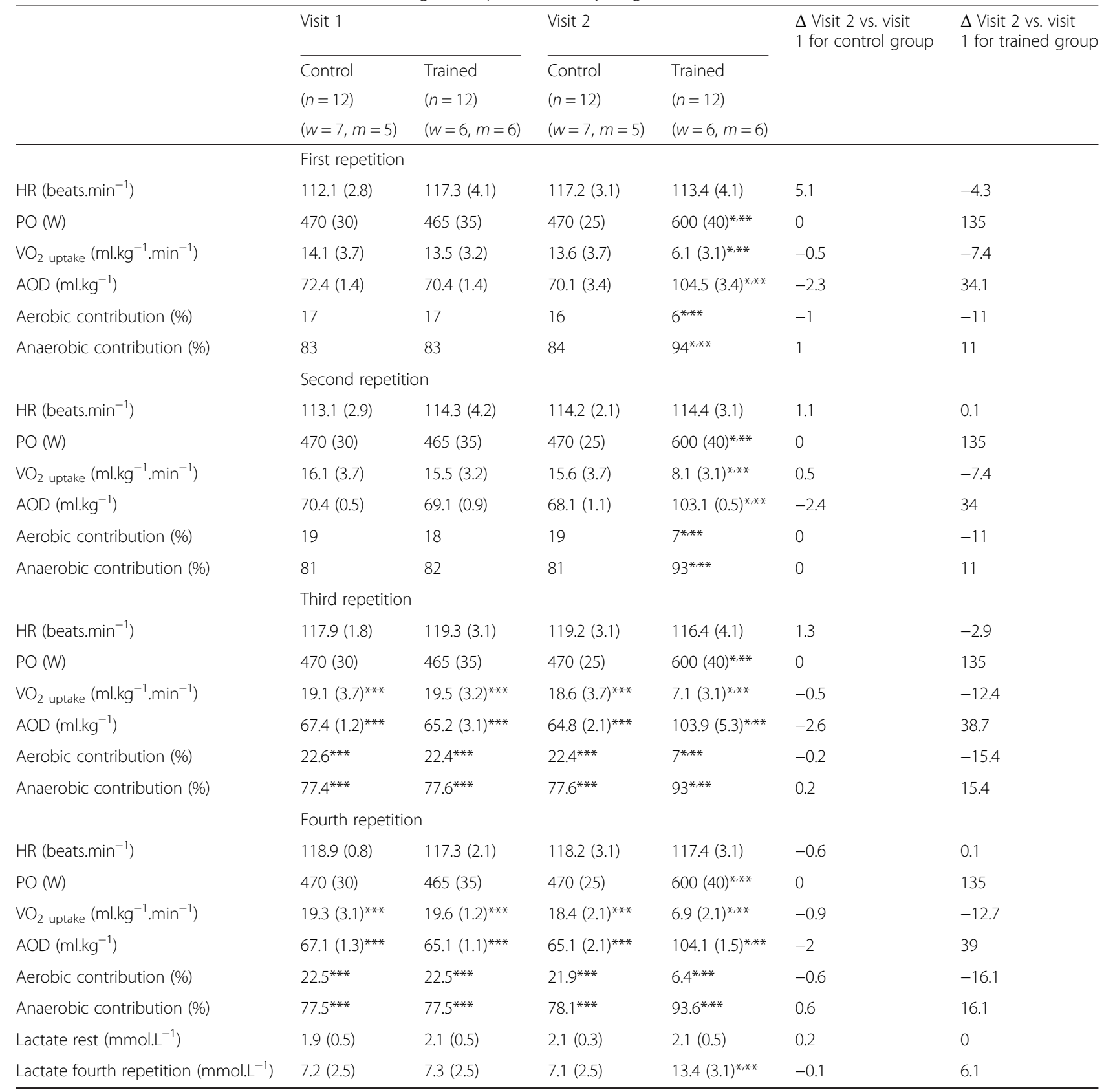

Values are expressed as the mean (standard deviation)

$w$ woman, $m$ men, $H R$ heart rate, $P O$ maximal power output developed during the $S C E, V O_{2}$ uptake oxygen uptake during repetition, $A O D$ accumulated oxygen, $\Delta$ variation between parameters assessed at visit 1 and visit 2

*Significant difference between groups $(p<0.01)$; ${ }^{* *}$ significant difference from visit $1(p<0.01)$; ***significant difference from the first repetition $(p<0.01)$

were accompanied by both greater AOD deficits and larger decreases in oxygen uptake during SCE repetitions. Our results revealed that for the same relative work (at maximal power output), HIT may be an appropriate approach for enhancing anaerobic capacity, reasonable given that this type of exercise training improves glycolytic activity [25].

The performances demonstrated by our experimental subjects during SCE satisfied the criteria for supramaximal testing. Indeed, the PO exhibited by our groups during SCE was much closer to their maximal power output values $\left(\mathrm{PO}_{\max }\right)$, which were obtained during the forcevelocity test $(p=0.1)$. Moreover, the PO exhibited by our groups did not differ during the fourth repetition. The AOD values obtained in our study were similar between the groups before HIT (74.4 vs. $\left.73.4 \mathrm{ml} . \mathrm{kg}^{-1}\right)$. Ten seconds of SCE may be considered a brief event during which subjects pedaled at maximal velocities against resistances 
determined during a force-velocity test [26, 27]. To meet the high ATP demand of SCE (e.g., adenosine diphosphate [ADP]), phosphorylation from $\mathrm{PCr}$ hydrolysis and glycolysis is necessary to overcome the oxygen deficit at the onset of exercise [28]. Several studies indicate that for events lasting 6-15 s, anaerobic contributions in sprinters are estimated to be $98 \%$ of these individuals' total energy expenditure [29]. Although AOD is a useful tool for determining anaerobic energy release [11], the present study demonstrated in the control group that the energy supply needed to perform $6 \mathrm{~s}$ during the first repetition of SCE results from anaerobic contributions $(\sim 83 \%)$, as well as a small aerobic supply $(\sim 15 \%)$. Therefore, contrary to the above findings, aerobic contributions to $6 \mathrm{~s}$ of SCE performance in obese adults represent a significant portion of the energy supply, which may result in lower rates of anaerobic glycolysis. The same tendency was also previously reported in normalweight subjects, suggesting that this incompatibility between anaerobic energy release and power output during supramaximal exercise is sometimes compensated for by the increased contributions of aerobic metabolism, as reflected by the increase in oxygen consumption [26]. It is likely that the relative contributions of different energy systems during repeated-sprint exercises depend on the exercise protocol (duration, number of repetitions, recovery duration, passive or active recovery) and training status. In the context of obesity, we also speculate that other factors such as reduced motor-unit activation [8], premature fatigue and reduced activation of type II muscle fibers [7] may affect the energy contribution profile. Unfortunately, none of these factors were assessed in the present study.

During the second, third, and fourth repetitions, there were decreases in anaerobic contributions, which became significant during the third and fourth SCE bouts. Conversely, aerobic energy contributions increased and reached a value of participation of approximately $22 \%$. We speculate that examinations of repeated-sprint exercises may offer supplement information concerning changes in muscle metabolism and other factors affecting performance. In the present study, there was an increase in aerobic solicitation in both the control group and the trained group before HIT, during the second, the third, and the fourth repetitions. Given that our exercise was a model of intermittent exercise with $2 \mathrm{~min}$ of recovery between each repetition, it may be that the synthesis capacity of muscle was modified by obesity. In normal-weight subjects, several studies have indicated that the resynthesis of substrates such as $\mathrm{PCr}$ depends on several factors (endurance fitness, training status) $[26,30]$, factors that may affect the energy profile of muscle during subsequent exercise. Despite the absence of data regarding muscle recovery rates during supramaximal exercise in the obese population, the probable impact of obesity on the capacity of muscle resynthesis cannot be ruled out as an explanation for our results.

Following HIT, there was an $11 \%$ reduction in calculated aerobic contributions to SCE, primarily as a result of an $11 \%$ increase in anaerobic energy contributions. Moreover, the lactate response to the fourth SCE bout increased significantly following HIT. In the present study, the lactate concentrations were used to compare the anaerobic potential processes between subjects during this type of exercise [31]. Our results demonstrated that anaerobic capacity may improve after only 6 weeks of HIT, reflecting enhancements in glycolysis activation. Anaerobic capacity responses to HIT have typically been assessed by measuring blood lactate levels in response to standardized exercise load or by measuring anaerobic performance via a Wingate test. A number of studies have demonstrated that HIT lasting between 2 and 15 weeks results in significant increases in anaerobic capacity, increases ranging between 5 and $28 \%$ in untrained males $[21,32,33]$, as well as in overweight and obese men [10].

The mechanism(s) underlying these adaptations are likely the result of skeletal muscle adaptations involving marked increases in skeletal muscle capacity for glycolytic enzyme content [34], increases in anaerobic fitness, and increased insulin sensitivity. Additionally, it should be noted that obesity does not appear to negatively affect intrinsic muscle contractility properties ("muscle quality") [35]. For Maffiuletti et al. [36], the presence of factors such as the handicapping effects of excess fat mass and impaired motor coordination may account for the poor physical performances of obese people of all ages. In the present study, a positive relationship between PO and both anaerobic contributions and lactate concentrations was noted. Concerning the lactate concentrations, which obtained via capillary blood samples using the LactatePro analyzer, higher values obtained after HIT may probably reflect amelioration in glycolysis activity in trained obese group. Although enhancements in anaerobic contributions were associated with $\mathrm{PO}$ enhancements, it is possible that the improvements in muscle coordination induced by HIT may also have contributed to $\mathrm{PO}$ and to lactate improvement.

From a clinical point of view, our results are valuable in understanding the interaction between intensity and energy contributions during high-intensity exercise. This study demonstrates that incompatibilities in energy balance during SCE bouts may reflect lower anaerobic contributions or poor skeletal muscle function in obese adults. This may, in turn, help clarify why obese people require high-intensity training as part of their multicomponent treatment for obesity.

The Wingate test (30 s of all-out sprint) has been the protocol utilized most often in the context of highintensity training. This protocol consists of 3 to $4 \mathrm{~min}$ of 
cycle exercise per session, and each session typically occurs three times per week. This protocol, although remarkably short in duration, is extremely rigorous, and subjects must tolerate significant discomfort. Our study is the first to demonstrate that 6 weeks of very short repeated bouts of SCE may improve muscle power, improvements associated with increased anaerobic system contributions. In spite of these adaptations, more studies are necessary in order to understand the effects of such protocols on other health parameters. Finally, although the cardiovascular and metabolic consequences of obesity have been studied extensively over the last two decades, less attention has been paid to investigating the impact of obesity on muscle-energetic interactions for a given exercise. The present study has forced us to consider muscle energy potential during exercise in order to describe the true energetic adaptations that occur during exercise and following a training program for obese individuals.

However, there are some limits in the current study. Firstly, the missing post-intervention measure in several metabolic (e.g., lactate) and hormonal (e.g., catecholamines) parameters, besides psychological variables, may allow us to better understand the effect of such exercise training on metabolic and hormonal adaptations. Secondly, the physical activity and dietary level $48 \mathrm{~h}$ prior to the test were not directly controlled, and then we cannot be sure if our instructions were fully respected. Finally, the results obtained in the present study included men and women in the same study group. Given that gender could have affected our results, further studies are needed to address this effect.

\section{Conclusions}

In conclusion, this study demonstrated the existence of a disparity in relative anaerobic and aerobic metabolism contributions to total energy release during supramaximal effort in obese adults, before and after HIT. Increased anaerobic contributions after HIT were associated with increased power output, although no changes in fat-free mass were observed. Such results highlight the importance of HIT in enhancing muscle tolerance to exercise by improving anaerobic capacity. Thus, the present findings provide an important first step towards an evidence-based approach for the utilization of HIT as a strategy for the obese sedentary population. However, the role of other training model (e.g., aerobic training) on improving several health parameters (e.g., cardiovascular and metabolic adaptations) cannot be excluded in the context of obesity management.

Finally, the evaluation of energy interactions during exercise must be examined in obese individuals.

\section{Competing Interests}

The authors declare that they have no competing interests.

\section{Authors' Contributions}

GJ: Conceived and desined the experiments, performed the experiments, analysed the data, wrote the paper and approved the final version. H-D I: Performed the experiments, corrected the paper and approved the final version. AP: Analysed the data, corrected the paper and approved the final version.

\section{Acknowledgements}

We are grateful for the cooperation and participation of the subjects. We also thank to Monique Dufour for her technical assistance.

This study was funded by the Faculty of Superior Studies and Research of the Université de Moncton.

Received: 5 September 2015 Accepted: 15 September 2015

Published online: 20 October 2015

\section{References}

1. Spencer MR, Gastin PB. Energy system contribution during 200- to 1500-m running in highly trained athletes. Med Sci Sports Exerc. 2001;33:157-62.

2. Zouhal H, Jabbour G, Jacob C, Duvigneau D, Botcazou M, Ben Abderrahaman $\mathrm{A}$, et al. Anaerobic and aerobic energy system contribution to 400-m flat and 400-m hurdles track running. J Strength Cond Res. 2010;24(9):2309-2315.

3. Vettor R, Macor C, Rossi E, Piemonte G, Federspil G. Impaired counterregulatory hormonal and metabolic response to exhaustive exercise in obese subjects. Acta Diabetol. 1997;34(2):61-6.

4. Zouhal H, Lemoine-Morel S, Mathieu ME, Casazza GA, Jabbour G. Catecholamines and obesity: effects of exercise and training. Sports Med. 2013;43(7):591-600.

5. Lazzer S, Boirie Y, Bitar A, Petit I, Meyer M, Vermorel M. Relationship between percentage of VO2max and type of physical activity in obese and non-obese adolescents. J Sports Med Phys Fitness. 2005;45(1):13-9.

6. Anderssen SA, Cooper AR, Sardinha LB, Harro M, Brage S, Andersen LB. Cardiorespiratory fitness is a strong predictor for clustering of cardiovascular disease risk factors in children independent of country, age and sex. Eur J Cardiovasc Dis Prev Rehabil. 2007;14:526-31.

7. Duché P, Ducher G, Lazzer S, Doré E, Tailhardat M, Bedu M. Peak power in obese and nonobese adolescents: effects of gender and braking force. Med Sci Sports Exerc. 2002;34(12):2072-8.

8. Blimkie CJ, Sale DG, Bar-Or O. Voluntary strength, evoked twitch contractile properties and motor unit activation of knee extensors in obese and nonobese adolescent males. Eur J Appl Physiol Occup Physiol. 1990;61(3-4):313-8.

9. Babraj JA, Vollard NBJ, Keast C, Guppy FM, Cottrell G, Timmons JA. Extremely short duration high intensity training substantially improves insulin action in young sedentary males. BMC Endocr Disord. 2009;9:3.

10. Whyte $L$, Gill JM, Cathcart AJ. Effect of 2 weeks of sprint interval training on health-related outcomes in sedentary overweight/obese men. Metabolism. 2010;59(10):1421-8.

11. Medbo Jl, Mohn AC, Tabata I, Bahr R, Vaage O, Sejersted OM. Anaerobic capacity determined by maximal accumulated $\mathrm{O} 2$ deficit. J Appl Physiol. 1988:64:50-60.

12. Adami A, Capelli C. Oxygen deficit during supramaximal cycling exercise in humans: a new estimation method. J Sports Med Phys Fitness. 2013;53(1):17-26.

13. Hirvonen J, Rehumen S, Rusko M, Harkönen M. Breakdown of high energy phosphate compounds and lactate accumulation during short supramaximal exercise. Eur J Appl Physiol. 1987;56:253-59.

14. Craig CL, Marshall AL, Sjostrom M, Bauman AE, Booth ML, Ainsworth BE, et al. International physical activity questionnaire: 12 - country reliability and validity. Med Sci Sports Exerc. 2003; 35: 1381-95.

15. Jabbour G, Lemoine-Morel S, Casazza GA, Hala Y, Moussa E, Zouhal H. Catecholamine response to exercise in obese, overweight, and lean adolescent boys. Med Sci Sports Exerc. 2011;43(3):408-15.

16. Canadian Guidelines for Body Weight Classification in Adults- Quick Reference Tool for Professionals [Cited 2014 Sept 20]. Available from: http://www.hc-sc.gc.ca/fn-an/nutrition/weights-poids/guide-ld-adult/ index-fra.php.

17. Spiro SG. Exercise testing in clinical medicine. Br J Dis Chest. 1977;71:145-72.

18. Ramsbottom R, Nevill AM, Nevill ME, Newport S, Williams C. Accumulated oxygen deficit and short-distance running performance. J Sports Sci. 1994;12(5):447-53. 
19. Woolford SM, Withers RT, Craig NP, Bourdon PC, Stanef T, McKenzie I. Effect of pedal cadence on the accumulated oxygen deficit, maximal aerobic power and blood lactate transition thresholds of high-performance junior endurance cyclists. Eur J Appl Physiol Occup Physiol. 1999;80(4):285-91.

20. Vandewalle HPG, Heller J, Monod H. Interests and limits of the speed-force relation in human. Sci Mov (in French). 1988;4:38-46.

21. Tabata I, Nishimura K, Kouzaki M, Hirai Y, Ogita F, Miyachi M, et al. Effects ofmoderate-intensity endurance and high-intensity intermittent training on anaerobic capacity and VO 2max. Med Sci Sports Exerc. 1996;28(10):1327-30.

22. Coyle EF. Very intense exercise-training is extremely potent and time efficient: a reminder. J Appl Physiol. 2005;98:1983-4.

23. Gibala MJ. High-intensity interval training: a time-efficient strategy for health promotion? Curr Sports Med Rep. 2007;6:211-3.

24. Gastin PB, Costill DL, Lawson DL, Krzeminski K, McConell GK. Accumulated oxygen deficit during supramaximal all-out and constant intensity exercise. Med Sci Sports Exerc. 1995;27(2):255-63.

25. Dawson B, Fitzsimons M, Green S, Goodman C, Carey M, Cole K. Changes in performance, muscle metabolites, enzymes and fiber types after short sprint training. Eur J Appl Physiol Occup Physiol. 1998;78:163-9.

26. Bogdanis GC, Nevill ME, Boobis LH, Lakomy HK. Contribution of phosphocreatine and aerobic metabolism to energy supply during repeated sprint exercise. J Appl Physiol. 1996;80(3):876-84.

27. Bogdanis GC, Nevill ME, Boobis LH, Lakomy HK, Spencer M, Bishop D, et al. Physiological and metabolic responses of repeated-sprint activities: specific to field-based team sports. Sports Med. 2005;35(12):1025-44.

28. Greenhaff PL, Campbell-O'Sullivan SP, Constantin-Teodosiu D, Poucher SM, Roberts PA, Timmons JA. An acetyl group deficit limits mitochondrial ATP production at the onset of exercise. Biochem Soc Trans. 2002;30(2):275-80

29. Fox EL, Mathews DK. Interval training: conditioning for sports and general fitness. Philadelphia: W. B. Saunders; 1974.

30. Gaitanos GC, Williams C C, Boobis LH, Brooks S. Human muscle metabolism during intermittent maximal exercise. J Appl Physiol. 1993;75:712-19.

31. Wasserman K, Hansen JE, Sue DY, Whipp BJ, Casaburi R. Principles of exercise testing and interpretation. Philadelphia: Lea \& Febiger; 1994

32. Medbo Jl, Burgers S. Effect of training on the anaerobic capacity. Med Sci Sports Exerc. 1990;22(4):501-7.

33. Ziemann E, Grzywacz T, Łuszczyk M, Laskowski R, Olek RA, Gibson AL. Aerobic and anaerobic changes with high-intensity interval training in active college-aged men. J Strength Cond Res. 2011;25(4):1104-12.

34. Nevill ME, Holmyard DJ, Hall GM, et al. Growth hormone responses to treadmill sprinting in sprint- and endurance-trained athletes. Eur J Appl Physiol Occup Physiol. 1996;72(5-6):460-7.

35. D'Hondt E, Deforche B, Vaeyens R, Vandorpe B, Vandendriessche J, Pion J, et al. Gross motor coordination in relation to weight status and age in 5- to 12-year-old boys and girls: a cross-sectional study. Int J Pediatr Obes. 2011;6:556-64.

36. Maffiuletti NA, Ratel S, Sartorio A, Vincent M. The impact of obesity on in vivo human skeletal muscle function. Curr Obes Rep. 2013;2:251-60.

\section{Submit your manuscript to a SpringerOpen ${ }^{\circ}$ journal and benefit from:}

- Convenient online submission

- Rigorous peer review

- Immediate publication on acceptance

- Open access: articles freely available online

- High visibility within the field

- Retaining the copyright to your article

Submit your next manuscript at $>$ springeropen.com 